\title{
Context Effects at Encoding and Judgment in Consumption Settings: The Role of Cognitive Resources
}

\author{
JOAN MEYERS-LEVY \\ ALICE M. TYBOUT*
}

\begin{abstract}
When consumers learn about a new product, cues in the surrounding context have been found to bias their response to the product in two ways. In some instances, judgments of the product are assimilated toward the affect or descriptive implications associated with the context, whereas in other circumstances, responses are contrasted with or adjusted away from the context. We examine how cognitive resources influence whether assimilation or contrast occurs and when such context effects are reflected in subsequent judgments. Building on a model developed by Martin and his colleagues, we propose that assimilation will occur spontaneously during encoding. Contrast will occur only when this contextual influence is viewed as inappropriate and efforts to partial out the context result in overcorrection. These encoding effects of context should be evident in later judgments when the nature of either the judgment task or consumers' predisposition toward effortful thought encourage retrieval of the contextencoded information. The results of two experiments support our predictions and lead to a modified version of Martin's model. In this model, the cognitive resources available at encoding determine the type of context effect and the cognitive resources at judgment determine whether the encoding effect of context will be reflected in product evaluations.
\end{abstract}

Consingento onsider a situation in which advertising will be used to introduce a new product into the marketplace. Suppose that the launch advertisements are scheduled to air during an episode of a prime-time drama that is likely to evoke negative feelings: an adorable child is stricken by a life-threatening disease. Should the introduction of the product be delayed so that advertising can be placed in a more uplifting episode, say, one in which the child is cured, or should the introduction proceed as originally planned? This scenario poses questions of whether and how the programming context may affect the encoding of information about the new product and, thereby, consumers' response to the product after the program-induced mood has dissipated.

The literature on context effects suggests that consumers often assimilate their responses toward the affect or descrip-

*Joan Meyers-Levy is an associate professor of marketing at the Graduate School of Business, University of Chicago, Chicago, IL 60637. Alice M. Tybout is the Harold T. Martin Professor of Marketing at the Graduate School of Management, Northwestern University, Evanston, IL 60208. This work was supported by the Bozell, Jacobs, Kenyon and Eckhardt Faculty Research Fund at the Graduate School of Business, University of Chicago. The authors thank the acting editor, the associate editor, and the $J C R$ reviewers for their numerous helpful comments on earlier drafts of this manuscript. tive implications associated with an accessible contextual cue (Allen and Janiszewski 1989; Herr 1989; Mathur and Chattopadhyay 1991; Meyers-Levy and Sternthal 1993; Schumann and Thorson 1990; Shimp, Stuart, and Engle 1991). This observation would seem to imply deferring the advertising launch until a more positive programming context can be insured. However, there have also been reports of a contrast effect in the literature (Herr 1989; Kamins, Marks, and Skinner 1991; Lynch, Chakravarti, and Mitra 1991; Meyers-Levy and Sternthal 1993). Here, there is a movement away from the implications of the context. Contrast implies that a program should be chosen that evokes negative rather than positive feelings.

An informed decision in our ad placement scenario requires a theoretical framework that anticipates when each type of context effect will occur. Two such frameworks exist that differ based on whether the focus is on the effect of the context during encoding (Martin 1986; Martin and Achee 1992; Martin, Seta, and Crelia 1990) or at judgment (Schwarz and Bless 1992). Because we examine a new product situation in which the context varies when information is encoded, we adopt a modified version of Martin's model as the starting point for our theorizing. Consideration of how this view is related to the context effects at judgment studied by Schwarz and Bless (1992) is deferred until the general discussion. 
Martin and his coworkers (Martin 1986; Martin and Achee 1992; Martin et al. 1990) propose that two factors determine whether evaluations exhibit an assimilation or a contrast effect. One factor is how appropriate the processor perceives the consideration of contextual data to be in judging a target object. The other factor is the cognitive resources that the processor is both able and willing to expend when forming a response.

These factors are incorporated into a model, represented schematically in Figure $1 a$. As a starting point, Martin's model assumes that when encoding information about a target item in an impression formation task, individuals generally assimilate their reactions toward accessible contextual cues. However, because they desire to provide a judgment that is free of extraneous influence and that is reflective of their genuine feelings about the target, individuals may subsequently assess the appropriateness of their initial reaction. When this occurs, if, on the one hand, the contextual information is accessed and is perceived to be an appropriate basis for forming a reaction, the assimilation effect will persist. On the other hand, if contextual information is considered to be inappropriate, the perceiver is expected to partial out or suppress the inappropriate information in their reaction to the target item. This process of suppression or partialling out is both imperfect and prone to overadjustment. Thus, a contrast effect often results. Because the contrast process is resource demanding, it is likely to occur only when substantial resources can be devoted to forming a reaction. In the absence of such effort, the assimilation effect noted earlier is likely to persist. The final stage in the model entails using the residual (adjusted) response as a probe cue in a second assessment of inappropriateness.

A study by Meyers-Levy and Sternthal (1993) illustrates such context effects as they may occur in a consumer setting. Subjects who varied in the cognitive effort they were likely to devote to processing viewed an ad for a new restaurant that, in passing, mentioned the previous occupant of the restaurant site. This prior occupant, which represented the contextual data, was either another restaurant or a clothing store that was regarded relatively positively or negatively. Results revealed that regardless of the level of cognitive effort subjects devoted to the task, when the prior occupant was another restaurant and thus, due to high category overlap, might be viewed as appropriate for use in judging the new restaurant, subjects assimilated the two pieces of information. Hence, subjects evaluated the new restaurant more favorably when the former occupant, a restaurant, was viewed more positively. However, when the prior occupant was a clothing store and, as a result of low category overlap, might be viewed as inappropriate for inclusion in reactions to the new restaurant, only subjects who applied limited cognitive effort to the task exhibited an assimilation effect. Indeed, those who engaged in more effortful processing contrasted the contextual cue with the stimulus information. As such, they evaluated the new restaurant more favorably when the clothing store was viewed less positively.

In the present research, we extend Martin's framework for understanding context effects. This entails examining how several factors that operate in consumer milieus but have not been previously investigated may affect (1) the cognitive resources that consumers are able to devote to contextual data during encoding and, thus, whether their reactions will reflect assimilation or contrast and (2) the cognitive resources that consumers are motivated to employ at judgment and, thus, whether the effect of the context at encoding will be reflected in their evaluations.

Particular attention is devoted to exploring the role of category information. As demonstrated in the MeyersLevy and Sternthal (1993) study discussed earlier, the degree of category or other semantic overlap between the context and the target object may affect whether contextual cues are viewed as an appropriate basis for judging the target stimulus. In addition, we propose that the task of determining the target's specific category membership may usurp cognitive resources that might otherwise be devoted to correcting for the influence of contextual information that is judged to be inappropriate. If this occurs, then preformed affect associated with the target stimulus category may provide an accessible, context-independent basis for evaluating the target. Whether or not judgments are based on such category affect or some other accessible heuristic is expected to depend on the amount of cognitive resources the type of judgment requires or one's predisposition toward engaging in effortful thought in formulating a response.

\section{LITERATURE REVIEW}

Meyers-Levy and Sternthal's research (1993) establishes the applicability of Martin et al.'s theory (Martin 1986; Martin and Achee 1992; Martin et al. 1990) to the consumer domain by demonstrating the predicted context effects. Yet like most other context effects research, this work overlooks several aspects of consumer settings that may influence the level of cognitive resources that can be devoted to contextual information during encoding and the cognitive resources that will be expended to retrieve the context-encoded information at judgment.

\section{The Linkage between the Context and the Target}

Studies of context effects that occur at encoding have employed procedures whereby the context stimulates activation of cognitions that are potentially relevant to the target information presented. For example, Meyers-Levy and Sternthal's (1993) contextual cue was a retail establishment that varied in its favorableness and had previously occupied the same location as the target establishment. Similarly, in his person impression studies, Martin (1986; Martin et al. 1990) employed contextual cues pertaining to the feelings and personality traits of people. 
FIGURE 1

MODELS OF THE EFFECT OF CONTEXT

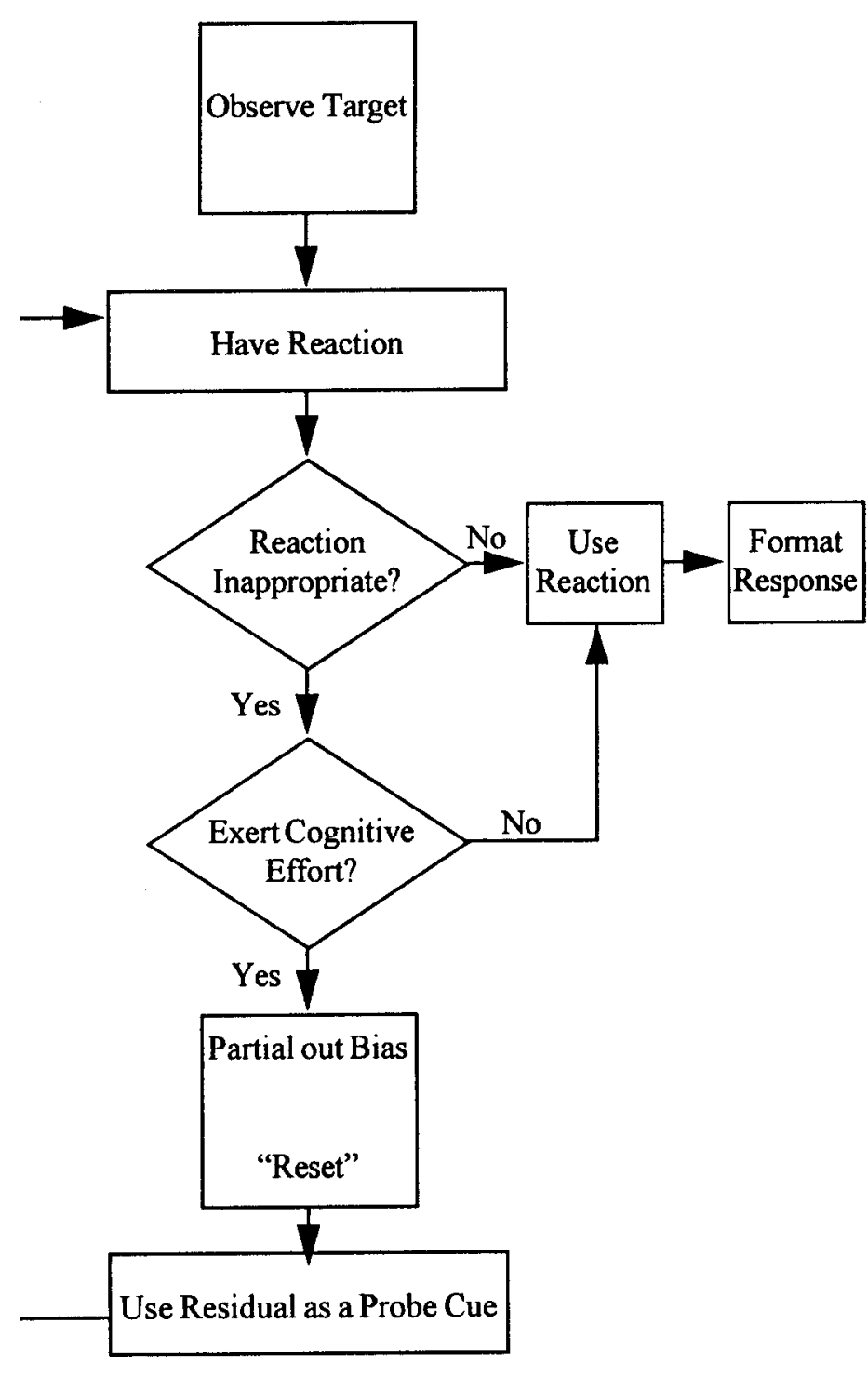

a

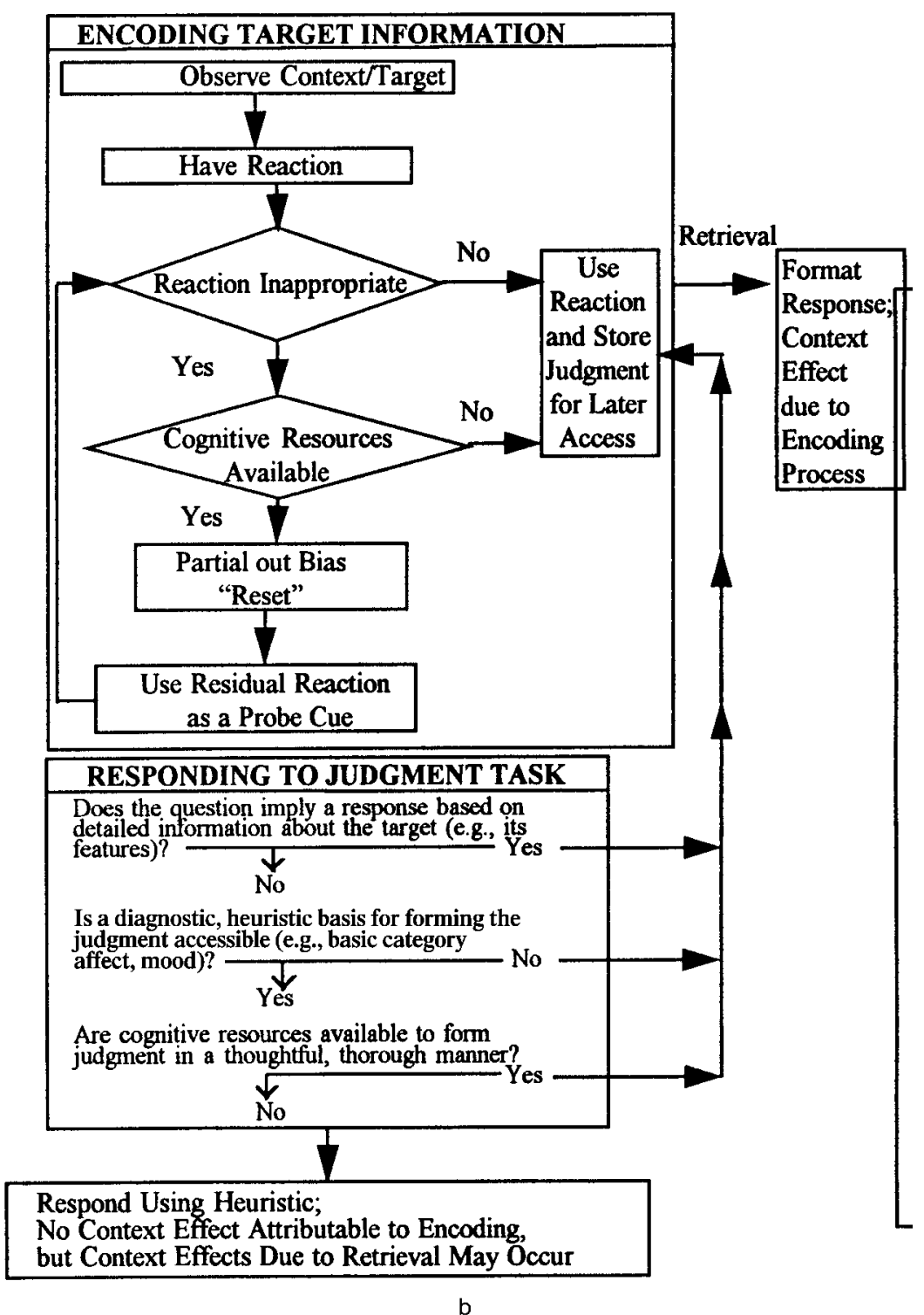

NotE._a, Schematic representation of Martin's set-reset model (from Martin et al. 1990, Fig. 2). b, Modified version of Martin's model. 
When such a conceptual linkage exists, the task of distinguishing between the context and the target would seem to be fairly resource demanding, potentially leading those who are not inclined to engage in extensive thought to conclude that the contextual information is an appropriate basis for response to the target. Consistent with this logic, assimilation has been the only context effect reported for individuals who are cognitive simplifiers (Meyers-Levy and Sternthal 1993) or who have a low need for cognition (Martin et al. 1990).

Context may also affect judgments even when it does not have a semantic connection to the target. This outcome has been observed in studies examining the effect of the mood induced by television programs and magazine articles on consumers' responses to advertisements placed in those media (e.g., Goldberg and Gorn 1987; Kamins et al. 1991; Yi 1990). The typical finding of assimilation to the context-induced mood would seem to be the result of this mood remaining accessible at judgment. Specifically, these studies have required consumers to evaluate concurrently both the program/article content and the embedded advertisements, thereby making context-induced feelings accessible when judgments of the advertisements were rendered (Schwarz and Bless 1993). Further, focus has centered on how the contextual mood affects evaluation of advertisements, and one's mood might reasonably be viewed as diagnostic for these judgments (Schwarz and Clore 1983).

It is less obvious that a contextual mood will influence the encoding of new product information and, thereby, have an effect on product evaluations that occur at a time when the contextual mood itself is no longer readily accessible. Research by Yi (1990) provides some insight into this issue. He conducted an experiment in which the context primed both a cognitive interpretation that was relevant to a focal attribute in a subsequently presented advertisement for a new product and a more general affective tone. Thus, subjects read a magazine article that pertained to either safety or fuel economy and that was framed in either a positive or a negative manner prior to evaluating a fictitious car for which the focal attribute was its large size. The findings revealed that the cognitive prime primarily affected the attitude toward the brand, whereas the affective prime primarily influenced attitude toward the ad. However, the influence of the affective prime may have been constrained by the presence of the cognitive prime, which could be used to infer a particular consequence of the car's large size.

In the absence of a cognitive prime, an affective prime might have a greater effect on information encoding than it did in Yi's (1990) study. This seems plausible because the associations generated in response to product attributes often are affect-laden, and the contextual mood might make affectively congruent associations more accessible. Thus, being in a negative mood upon learning that a new car is unusually large might generally predispose consumers toward unfavorable associations to this feature (e.g., fuel inefficient, difficult to park), while being in a positive mood might encourage more favorable interpretations (e.g., safe, comfortable ride). We explore this possibility by examining a situation in which the context only primes a general mood that lacks any particular meaning-based connection to the new product information.

Although a meaning-based linkage may not be required for the context to bias the encoding of product information, the absence of a such a linkage may influence the type of context effect that occurs. Specifically, when there is no semantic connection between the context and target, few cognitive resources may be required to detect that the contextual bias is inappropriate. Thus, even those consumers who are not inclined to engage in effortful thought may be motivated to partial out the effect of the context in order to determine their "true" response.

\section{The Effect of Categorization Task Demands on Correction for Inappropriate Contextual Influence}

Although all consumers may be motivated to partial out the influence of contextual mood, their success in doing so may be constrained by other aspects of the encoding task that draw on the available cognitive resources. In particular, when learning about an unfamiliar product, the ease with which the product can be categorized as a member of a meaningful product category may affect the resources that can be devoted to correcting for contextual influence. Rosch (1978) provides evidence that individuals naturally strive to categorize objects at a so-called basic level, which is particularly informative because it maximizes the ratio of attributes shared by members of the category relative to attributes distinct to members of a category. Sujan and Dekleva (1987) suggest that product types, such as soft drinks and fruit juices, correspond to Rosch's basic-level object categories.

In prior studies investigating context effects at encoding, basic-level category information has generally been lacking. Rather, the target object has been represented as a member of a broad, ill-defined category such as "a person'" (Martin 1986; Martin et al. 1990) or "'a new restaurant', (Meyers-Levy and Sternthal 1993). As a result, it is likely that consumers' categorization efforts have usurped resources that otherwise might be devoted to correcting judgments for inappropriate contextual influence, thereby generally encouraging assimilation effects.

By contrast, in actual consumer settings the product type is often clearly and immediately identified. Thus, more cognitive resources should be available for partialling out any inappropriate contextual influence. The implication is that a contrast effect may be more likely to emerge when specific category information is provided, whereas assimilation may be more likely to occur when such information is absent. 


\section{Observing the Encoding Effect of Context at Judgment}

In everyday settings by the time consumers express an evaluation of a product, data that are independent of the encoding context may be equally or more accessible than the information presented in an initial product description (advertisement). For example, if a new product claims or can be inferred to hold membership in an established, specific product category (e.g., soft drink), stored affect toward this category may be activated. Further, if advertising is followed by product sampling, this experience may provide sensory data that are sufficiently removed from the original context to be unaffected by it.

To date, the effect of such context-independent information has not been investigated. Two factors that seem likely to moderate consumers' motivation to retrieve the context-encoded product information when context-independent information that is potentially diagnostic is accessible are (1) the type of judgment requested and (2) consumers' predisposition to engage in effortful thought.

When asked to assess the level of well-specified product features (e.g., whether a new product is healthy or has a long shelf life), consumers may view information in the product description as uniquely diagnostic and, thereby, be motivated to devote cognitive resources to retrieving this information as a basis for their judgments (Park and Hastak 1994). Thus, context effects attributable to encoding are likely to occur for such feature judgments independent of the accessibility of other context-independent information and irrespective of consumers' general inclination toward effortful thought.

By contrast, more personal, overall affective evaluations (e.g., good/bad, like/dislike) may be viewed as not requiring retrieval of the detailed product description (i.e., other accessible information may be viewed as sufficiently diagnostic; Feldman and Lynch 1988). Instead, consumers' predisposition toward effortful thought may determine whether information in the product description is retrieved. Consumers who strive to be thoughtful and thorough in their evaluations may form their judgments in the manner implied by traditional models of attitude formation (e.g., Bettman, Capon, and Lutz 1975; Fishbein and Ajzen 1975). Specifically, they may retrieve several relevant product features, identify or infer the value associated with each feature, and both weigh and integrate all this information into an overall affective evaluation. If this occurs, then the influence of the context at encoding may be heightened because multiple features, each of which could be biased by the context during encoding, would be included in the overall judgment. Of course, this effortful approach to evaluation could also encompass other accessible information that is considered to be diagnostic.

However, consumers who are not predisposed to engage in such effortful processing may adopt a simpler, heuristic approach to forming their overall evaluations. They may base their judgments on accessible, preformed affect toward the product category (Fiske and Neuberg 1990; Simmons, Bickart, and Lynch 1993) or on feelings engendered by prior experience with the product or with other similar products. Alternatively, if such consumers truly wish to minimize their effort, they might base their evaluations on their general affective default value, which has been found to be moderately positive (Kaplan 1976; Markus and Zajonc 1985). In either case, effects of the context at encoding should be weakened or absent altogether for consumers who truly are disinclined to employ effortful processing, as they would be based on contextindependent data.

Interestingly, the pattern of data reported in MeyersLevy and Sternthal's (1993) work is congenial with the preceding interpretation of how both consumers' willingness to engage in effortful thought and the type of judgment requested may influence the observation of encoding effects on later judgment. They found that when consumers were predisposed toward effortful processing, the predicted effects of context reached conventional levels of significance $(p$ 's $<.05)$ regardless of the type of judgment requested. However, when consumers were inclined to minimize cognitive effort, such effects were significant on feature judgments $(p<.05)$, but they were only marginally significant on overall evaluations $(p<.10)$. Presumably those who wished to minimize their effort made greater use of context-independent information (e.g., their general disposition toward new restaurants and/or their default affect) when forming their overall evaluations versus their feature judgments. In fact, if this research had not employed a procedure that both enhanced the accessibility of the context (by incorporating it in the service description) and minimized the accessibility of contextindependent information (e.g., the specific type of restaurant was never identified, and no personal experience was provided), context effects might have been absent entirely on the overall evaluations of cognitive simplifiers.

In summary, factors present in many natural consumption settings suggest several modifications to Martin's model, which are represented in Figure $1 b$. First, the absence of a semantic linkage between the contextual data and the target product may reduce the cognitive resources required to detect the inappropriateness of the contextual influence. As a result, even consumers who are not naturally inclined to engage in effortful thought may recognize that their initial reactions or feature memories should be adjusted for this contextual influence. Second, the degree to which such adjustments are undertaken should be influenced by the extent to which product categorization usurps a sizable portion of the cognitive resources available during encoding. When such categorization is relatively resource demanding, the resources at one's disposal may be inadequate to allow consumers to correct for inappropriate contextual influence, and assimilation may persist. However, when categorization requires little effort, sufficient resources should be available for correction, and a contrast effect (due to overadjustment) may occur. Finally, although the context may bias encoding of new 
product information, whether this information will be retrieved at judgment will be determined by the cognitive resources that consumers are motivated to devote to formatting a response. Context effects attributable to encoding are likely to be absent at judgment when $(a)$ people have access to seemingly diagnostic context-independent data, such as the target product's specific category membership or prior experience with the product or like ones, $(b)$ individuals are not predisposed toward effortful processing, and $(c)$ the judgment requested pertains to matters of personal opinion such that it can be formed without directly retrieving data about specific product features. In such instances, people are likely to render their judgments in a relatively heuristic manner, relying on accessible data that are independent of the encoding context.

We report two experiments that test these hypotheses under conditions intended to represent consumer settings in which engaging contextual information (a television show or a news story) is interrupted by the presentation of information about a new product (an advertisement), and consumers subsequently have an opportunity to sample the product. The first experiment examines context effects on overall affective evaluations as a function of the resources needed to resolve ambiguity concerning the product's precise (basic-level) category membership and subjects' predisposition to engage in effortful thought (as assessed by a need-for-cognition scale). The second experiment replicates and extends on the first by employing contextual data that are more similar to those that often are encountered by consumers, varying the resources needed to categorize the product in an alternative manner and examining the encoding effect of contextual data on both overall affective evaluations and specific feature judgments.

\section{EXPERIMENT 1}

\section{Method}

Subjects. Eighty-one male and female students at a West Coast university completed the study in large groups. Each participant received five dollars as compensation.

Procedure. Subjects were informed that they would be participating in two market research studies for two different companies. The first study, described as an examination of people's ability to empathize with others, exposed subjects to contextual data that prompted either positive or negative thoughts. Specifically, subjects were randomly distributed booklets containing either eight positive or eight negative statements similar to those used by Martin (1986; e.g., "When I'm feeling this good [bad], even the smallest things in life are a great pleasure [pain]''). Participants were told that they would be paced through the eight statements, each purportedly written by another person. Their task was to read each statement and express in their own words the feelings of the other person.
Following this procedure, all subjects were interrupted and informed that it was necessary to move on to the second study. This task interruption procedure, which was patterned after one employed by Martin (1986, experiment 3), was intended to encourage individuals to engage in rumination about the statements such that their contextinduced affective thoughts would be accessible when they encoded the new product description presented at the start of the second study. Such a procedure is analogous to media contexts in which ads appear in editorial or program content that is thought provoking and affect laden.

The second study followed immediately. It was described as a new-product evaluation and taste test in which subjects would read a description of a new beverage, evaluate the product, taste a sample of it, and then reevaluate the drink. The beverage description was then presented, and it portrayed the product as a nonalcoholic, vitamin-laden, carbonated drink with a slightly sweet but tart taste. The drink purportedly stayed fresh in the refrigerator, was an appropriate accompaniment for snacks or meals, and was dispensed in cans and bottles. This product description varied in its consistency with the category in which the product claimed membership, which was either the soft drink or the fruit juice category. This was done to manipulate the ease of and thus the level of cognitive resources required to categorize the product at the basic level. According to previous research, categorization uses fewer resources when a product's attributes are congruent with or match a product category that is evoked (Meyers-Levy and Tybout 1989).

Specifically, all subjects read a product description that pretesting had indicated was more compatible with the soft drink than the fruit juice category $(\bar{X}=4.87$ vs. 3.53 on seven-point scales labeled not at all/extremely similar to a soft drink or fruit juice; $t(14)=2.23, p<.04)$. For half of the subjects, the beverage was labeled as a soft drink, while for the remaining subjects it was labeled as a fruit juice. Hence, when the category label and product description matched (soft drink condition), categorization was expected to require few resources. When this label and the description were mismatched (fruit juice condition), categorization should require more resources.

After reading the beverage description, subjects evaluated the drink on seven dimensions using seven-point semantic differential scales. Twenty-six pretest subjects had categorized these dimensions as more appropriate for expressing their summary or overall feelings about a beverage ( 79 percent) than for describing specific beverage features ( 21 percent). These overall affective evaluation items examined subjects' willingness to try the beverage, the product's benefits, such as its appeal and bad/ good taste, and the likelihood that the product was satisfying, desirable, refreshing, and of high quality.

Next, subjects completed a need-for-cognition scale (Cacioppo and Petty 1982) and various demographic questions. Product sampling followed. All subjects tasted a sample of Orangina, a sparsely distributed natural soft drink that contains real fruit juice. In a blind taste test 
performed earlier, this brand was found to evoke a relatively neutral response. Finally, subjects evaluated the beverage posttaste on the same scales used at pretaste.

Hence, the study employed a $2 \times 2 \times 2$ factorial design. Two factors represented between-subjects, randomly assigned conditions: favorableness of the contextual data (positive, negative) and the resources required for product categorization (few - soft drink category, many-fruit juice category). The third factor was the measured needfor-cognition (NFC) variable, which assessed subjects' predisposition to engage in thoughtful processing.

\section{Hypotheses}

As outlined earlier, we began with Martin's assumption that reactions are spontaneously assimilated to the context during encoding. Further, we have proposed that in situations such as the one we examine, in which the context lacks any semantic connection with the target, little effort is required to detect that any contextual influence is inappropriate. Thus, all subjects should be motivated to partial the contextual influence out of their reactions at encoding. However, the resources that are required to resolve ambiguity regarding the product's category membership are expected to moderate whether correction is actually able to occur. When the categorization task requires few resources, subjects are anticipated to possess sufficient resources to partial out contextual influence, but overadjustment in this process may produce a contrast effect. When categorization is more resource demanding, subjects are expected to lack the resources to correct for contextual influence, and an assimilation effect is predicted.

Although the foregoing effects of context that take place at encoding are anticipated to occur for all subjects, they are only expected to be manifested in this study on the overall evaluations of individuals whose need for cognition is high and who thereby are motivated to retrieve the detailed product information at judgment. These encoding effects of context are predicted to be absent for individuals whose need for cognition is low because seemingly diagnostic, heuristic bases for forming such overall evaluations are accessible. Taken together, our predictions imply that a three-way interaction of the favorableness of the contextual data, the resources required for categorization, and subjects' NFC should emerge on overall evaluations.

\section{Results and Discussion}

Subjects' scores ranged from 127 to 299 on the NFC scale $(\alpha=.89)$. Subjects were classified as high or low in NFC on the basis of a median split. Factor analysis confirmed that the seven evaluation measures tapped a single underlying dimension. Accordingly, these measures were averaged to form indices of both pretaste and posttaste overall affective evaluations $(\alpha=.85$ and .95 , respectively). Treatment means are reported in Table 1. Because treatment effects were absent on the pretaste evaluation index, further mention of this variable is deferred until the results of experiment 2 are presented.

Examination of posttaste overall affective evaluations revealed the anticipated three-way interaction of favorableness of the contextual data, resources required for categorization, and $\operatorname{NFC}(F(1,71)=11.89, p<.01)$. Follow-up analyses offer support for our hypotheses. Subjects who were low in NFC produced moderately positive evaluations, irrespective of the experimental treatments $(F<1)$. Although the context presumably affected the encoding of detailed product information, these consumers apparently relied on more accessible, context-independent information (e.g., preformed affect toward the basiclevel category or prior experience) when expressing their overall affective evaluations.

By contrast, subjects who were high in NFC produced overall evaluations that varied depending on the contextual data they received and the resources required for product categorization $(F(1,71)=17.85, p<.05)$. A contrast effect emerged when categorization required few resources (soft drink condition; $F(1,71)=4.03, p<.05$ ), whereas an assimilation effect emerged when categorization was more resource demanding (fruit juice condition; $F(1,71)=16.59, p<.01)$. Thus, it appears that high NFC individuals retrieved and used the context-encoded information as a basis for their evaluations despite the accessibility of other, potentially diagnostic context-independent information.

Although these outcomes are consistent with our theorizing, the absence of any significant effect for low NFC individuals is problematic. Instead of lacking the motivation to retrieve context-encoded product information, it is plausible that low NFC individuals were unaffected by the context at encoding. To rule out this possibility, it is important to demonstrate that the judgments of low NFC individuals reflect the same influence of the contextual cues and cognitive resources as high NFC individuals when they are motivated to retrieve the product information. A second experiment, which included specific feature judgments, was undertaken to address this issue and to establish the robustness of the findings observed in experiment 1.

Based on the reasoning outlined earlier, we anticipated that responding to specific feature judgments would prompt both high and low NFC subjects to retrieve the context-encoded information provided in the product description. As a result, a two-way interaction of the favorableness of the contextual data and resources required for categorization was predicted for feature judgments. When categorization required few resources, consumers were expected to possess sufficient resources to partial out the contextual influence, but overadjustment in this process might produce a contrast effect. When categorization was more resource demanding, consumers should lack the resources required to correct for the contextual influence, and an assimilation effect was anticipated. In contrast, 
TABLE 1

EXPERIMENT 1 TREATMENT MEANS

\begin{tabular}{|c|c|c|c|c|}
\hline \multirow[b]{2}{*}{ Dependent variable } & \multicolumn{2}{|c|}{ Many resources for categorization } & \multicolumn{2}{|c|}{ Few resources for categorization } \\
\hline & Positive context & Negative context & Positive context & Negative context \\
\hline \multicolumn{5}{|c|}{ Pretaste affective evaluations: } \\
\hline Low NFC & 5.39 & 5.52 & 5.48 & 5.12 \\
\hline High NFC & 4.99 & 4.94 & 4.73 & 4.79 \\
\hline \multicolumn{5}{|c|}{ Posttaste affective evaluations: } \\
\hline Low NFC & 4.32 & 4.74 & 5.52 & 5.02 \\
\hline High NFC & 5.27 & 2.88 & 3.86 & 5.17 \\
\hline
\end{tabular}

NotE. - NFC $=$ need for cognition.

low NFC individuals were expected to view accessible, context-independent information as sufficiently diagnostic for their more personal, overall affective judgments. As a result, it was anticipated that NFC would moderate retrieval of the context-encoded information when overall affective evaluations were requested. Thus, the three-way interaction reported in the present study should be replicated for such evaluations.

\section{EXPERIMENT 2}

\section{Method}

Subjects. Subjects consisted of 77 female undergraduates at a midwestern university who were recruited to participate in two market research studies. Five to 12 people participated in each session, and each person was paid $\$ 5$ for their cooperation.

Procedure. The basic procedure employed in this study was similar to that used in experiment 1 , although several important changes were made. One was that the eight positive or negative statements used to activate affective thoughts in experiment 1 were replaced by eight positive or negative news stories that were similar to those that often appear in actual newspaper or magazine contexts. Pretesting indicated that these news stories were equivalent and near the midpoint on dimensions of writing clarity and interest level, but the positive news stories were more uplifting and happy than were the negative stories $(F(1,24)=55.88, p<.001$; see the Appendix for sample stories, and see Yi [1990] for another example of news stories used to activate such affective thoughts). As in experiment 1, subjects responded to the feelings expressed in four of these news stories and then were interrupted and told to move on to the second study.

The manipulation used to vary the resources required to categorize the product was also altered. This entailed slightly modifying the previously employed beverage description to adapt to changes that had occurred in the soft drink category (e.g., the emergence of new age, juiceinfused beverages) and to make the description equally fitting for a soft drink or a fruit juice. Thus, the beverage was described as having the following characteristics: slightly sweet, best served chilled, tart, available in convenience food stores, carbonated, tingly, quickly absorbed into one's bloodstream, vitamin and calcium enriched, appropriate for snacks, priced at $\$ .47$ per 12-ounce serving, high in preservatives, available in cans and bottles, and appropriate for people with a zest for life. Fourteen pretest subjects who read the modified product description rated it on a seven-point scale as equally likely to be a soft drink $(\bar{X}=4.86)$ or fruit juice $(\bar{X}=4.36 ; p>.42)$. Further, all references to the beverage as either a soft drink or a fruit juice were removed from the description. Instead, just prior to reading the product description, the resources required to categorize the product were varied by presenting subjects with consensual data that pertained to the product's specific category membership. Half of the subjects, for whom categorization was intended to require few resources, were told that in a survey of 1,000 people who were asked how best to describe the product in question, 90 percent felt that the beverage should be described as a fruit juice, whereas only 10 percent felt that it should be classified as a soft drink. The remaining subjects, for whom categorizing the product conclusively was intended to be more resource demanding, were told that 50 percent of survey participants felt the beverage should be described as a soft drink and 50 percent felt it should be called a fruit juice.

A third change introduced in experiment 2 entailed the addition of items asking subjects to judge specific features of the product. Hence, interspersed among the same seven overall affective scale items used in experiment 1 were new items that pertained to specific product features discussed in the ad. These feature items ascertained how likely it was that the product was nutritious and healthy, tasted tangy, had a long shelf life, and would spoil quickly. ${ }^{1}$ Pretest subjects categorized these items as more appropriate for describing beverage features than for ex-

\footnotetext{
${ }^{1}$ Three additional measures (bitterness, vivaciousness, and effervescence) that were intended to assess product features were administered but were deleted from further analysis when factor analysis revealed that they cross-loaded with the overall evaluation items.
} 
pressing summary or overall feelings (on average, 80 percent of the 26 pretest subjects categorized these items as describing such features vs. 20 percent who categorized them as expressing summary feelings).

The only other procedural changes were the inclusion of thought-listing and recall tasks (discussed briefly in the general discussion), the use of an abbreviated 18item version of the NFC scale (Cacioppo, Petty, and Kao 1984), and the use of a different, little-known brand (called Boomerang) of flavored sparkling water containing pure fruit juice as the sampled product. As in the first study, this product also evoked a relatively neutral response in a blind taste test.

Hence, experiment 2 employed a $2 \times 2 \times 2 \times 2$ factorial design that included three of the same or similar factors employed in the previous study: favorableness of the contextual data (positive, negative) the resources required for product categorization (high consensus, low consensus), and need for cognition (NFC). The final factor was a repeated measure of judgment type (overall affective evaluations vs. feature judgments).

\section{Results and Discussion}

Subjects' scores on the NFC scale ranged from 45 to 153. Subjects were classified as high or low in NFC on the basis of a median split $(\alpha=.88)$. Factor analysis of the 12 judgment items revealed that the seven overall affective items loaded on a single factor and the five feature items loaded on two factors representing different underlying dimensions of the new product. Specifically, one feature factor comprised three items related to freshness (stays fresh long, tangy, and shelf life) and the second feature factor comprised two items related to health (healthy, nutritious). Because our theorizing pertains only to the distinction between feature judgments and overall affective evaluations and not to differences among feature judgments, and because analyses of separate indices representing each of the feature factors revealed the same pattern of outcomes, all five feature items were averaged to form a single feature index at pretaste and at posttaste $\left(\alpha=.43\right.$ and .73 , respectively). ${ }^{2}$ Pretaste and posttaste indices were also created for overall evaluations $(\alpha=.86$, and .96, respectively). These indices were analyzed using the full four-factor design identified previously, and we discuss only the highest order effects. ${ }^{3}$ Treatment means

\footnotetext{
${ }^{2}$ Separate analyses of a freshness index, created by averaging the three items loading on this factor, and a health index, created by averaging the two items loading on this factor, revealed that the predicted two-way interaction of the favorableness of contextual data and the resources required for categorization was significant for both measures $(F(1,68)$ $=4.06, p<.05 ; F(1,68)=3.86, p<.05$, respectively). No other interactions were significant for either index.

${ }^{3}$ Outcomes comparable to those reported here emerged on both overall affective evaluations and feature judgments when, using regression, NFC was treated as a continuous variable. Specifically, the three-way interaction of contextual data, resources required for categorization, and NFC remained significant for overall affective evaluations $(t=-2.76$, $p<.01)$, but the two-way interaction of contextual data and resources
}

are reported in Table 2. As in experiment 1, treatment effects were absent at pretaste (all $p$ 's $>.16$ ).

Analysis of posttaste product judgments revealed a four-way interaction of favorableness of contextual data, resources required for product categorization, NFC, and type of judgment $(F(1,68)=4.84, p<.03)$. Further examination of this effect revealed that, as expected, overall affective evaluations exhibited the same three-way interaction of favorableness of contextual data, resources required for product categorization, and NFC observed in experiment 1 . As in that study, subjects who were low in NFC evaluated the product in a moderately positive manner, irrespective of variations in the other two treatments $(F<1)$. However, the overall evaluations of subjects who were high in NFC revealed context effects that varied depending on the resources required for product categorization $(F(1,68)=8.71, p<.01)$. A contrast effect emerged when categorization required few resources (high consensus; $F(1,68)=3.57, p<.06$ ), whereas an assimilation effect emerged when categorization was more resource demanding (low consensus; $F(1,68$ ) $=9.09, p<.01)$.

At the same time, analysis for specific feature judgments revealed only the anticipated two-way interaction of favorableness of contextual data and resources required for categorization $(F(1,69)=9.73, p<.01)$. Such feature judgments displayed a contrast effect when product categorization required few resources (high consensus; $F(1,68)=3.86, p<.05)$ and an assimilation effect when categorization was more resource demanding (low consensus; $F(1,69)=5.49, p<.02)$, irrespective of subjects' NFC.

Thus, these findings are consistent with our hypotheses and support the modified view of Martin's model represented in Figure $1 b$. The predicted presence and absence of context effects observed on feature versus overall affective judgments is consistent with the view that because of their willingness to engage in effortful thought, high NFC subjects retrieved the product information that was influenced by the context during encoding irrespective of the type of judgment requested. By contrast, low NFC individuals were sensitive to the type of judgment requested. They exhibited context effects when feature judgments were requested, as these judgments presumably prompted retrieval of specific product features that were mentioned in the product description and encoded in a context-biased manner. Yet context effects were absent on overall affective evaluations that were more amenable to determination via heuristics based on accessible, context-independent data.

Although the absence of effects on pretaste measures in this experiment as well as in experiment 1 is not rele-

required for categorization for feature judgments remained unqualified by NFC $(t<1)$. A similar analysis performed for experiment 1 provided convergent evidence for the three-way interaction reported in that study $(t=-3.53, p<.01)$. 
TABLE 2

EXPERIMENT 2 TREATMENT MEANS FOR EVALUATION MEASURES

\begin{tabular}{|c|c|c|c|c|}
\hline \multirow[b]{2}{*}{ Dependent measure } & \multicolumn{2}{|c|}{ Many resources for categorization } & \multicolumn{2}{|c|}{ Few resources for categorization } \\
\hline & Positive context & Negative context & Positive context & Negative context \\
\hline \multicolumn{5}{|c|}{ Pretaste overall evaluations: } \\
\hline Low NFC & 5.45 & 4.71 & 5.44 & 5.42 \\
\hline High NFC & 5.33 & 5.14 & 5.40 & 5.77 \\
\hline \multicolumn{5}{|c|}{ Posttaste overall evaluations: } \\
\hline Low NFC & 4.94 & 4.87 & 5.21 & 4.81 \\
\hline High NFC & 4.90 & 2.43 & 4.10 & 5.23 \\
\hline \multicolumn{5}{|c|}{ Pretaste feature judgments: } \\
\hline Low NFC & 5.00 & 5.08 & 5.14 & 5.00 \\
\hline High NFC & 4.86 & 4.91 & 5.13 & 5.33 \\
\hline \multicolumn{5}{|c|}{ Posttaste feature judgments: } \\
\hline Low NFC & 5.34 & 4.33 & 4.26 & 4.73 \\
\hline High NFC & 4.84 & 3.80 & 4.13 & 5.25 \\
\hline
\end{tabular}

NOTE. $-\mathrm{NFC}=$ need for cognition

vant to our theorizing regarding context effects, it does merit some discussion. After all, pretaste measures were taken in closer proximity to the independent variables, and they were taken before actual product experience could offer an alternative and potentially context-independent basis for judgment.

There is precedence for effects emerging at posttaste but not pretaste in several other studies that employed procedures similar to ours (e.g., Meyers-Levy and Tybout 1989; Scott and Yalch 1978). This pattern is thought to emerge because consumers are often reluctant to express their views when they are based on data of questionable credibility and/or diagnosticity (e.g., descriptive information presumably provided by the product manufacturer) and they know that additional experiential or sensory data will be forthcoming (cf. Hoch and Ha 1986). Thus, although individuals presumably do form tentative judgments prior to product sampling that parallel those observed at posttaste, they may refrain from revealing those judgments until they can be tested and confirmed on the basis of the sensory data.

Two observations support such an interpretation of our findings. One is the finding that the same three-way interaction of favorableness of the contextual data, resources required for product categorization, and NFC that emerged on overall affective judgments was marginally significant on the positive thoughts subjects generated in a thought-listing task administered prior to sampling the product in experiment $2(F(1,68)=3.75, p=.06)$. Replicating the effects obtained on overall evaluations, the number of positive thoughts elicited by low NFC subjects was unaffected by the experimental treatment $(F<1)$, whereas the number of such thoughts produced by high NFC subjects exhibited a contrast effect when categorization required few resources (i.e., high consensus; $F(1$, $68)=5.07, p<.01)$ and an assimilation effect when categorization was more resource demanding (i.e., low consensus $(F(1,68)=7.14, p<.01)$. The fact that these outcomes were observed on this measure taken after subjects received the contextual and stimulus information but prior to sampling the product is consistent with the contention that subjects indeed formed but were unwilling to express their context-influenced reactions prior to testing them on the sensory data.

Additional support for this interpretation of our findings was obtained in a follow-up study that we conducted. This study was nearly identical to the present study except that all subjects were volunteers from a pool of graduate students, who tended to score high in NFC, and all were informed that they were participating in sessions where respondents would receive descriptive information about the product but would not be tasting it. Consistent with the view that subjects formed context-influenced hypotheses about the product prior to tasting it but refrained from expressing such views because they anticipated obtaining additional relevant (sensory) data in experiments 1 and 2 , subjects in this follow-up study, who did not expect and did not obtain such data, produced overall evaluations that were sensitive to both the favorableness of the contextual data and the resources required for product categorization $(F(1,48)=6.69, p<.01)$. Overall evaluations were contrasted with the affect associated with the context when few resources were required for categorization (high consensus; $F(1,48)=3.41, p<.07)$, while they were assimilated with such affect when categorization required many resources $(F(1,48)=3.28, p<.08) .{ }^{4}$ Thus, it appears that the context effects we obtain at posttaste may

${ }^{4}$ Examination of an aggregate feature index in this study failed to reveal significant effects. Although the feature items related to freshness followed the same general pattern that was observed for overall evaluations, the items related to health exhibited the inverse of this pattern. This latter outcome may be attributable to different inference processes occurring when subjects are aware that they will be unable to confirm their hypotheses through product experience. 
be evident at pretaste when consumers do not anticipate receiving additional, potentially diagnostic data.

\section{GENERAL DISCUSSION}

Our theorizing builds on Martin's model of context effects that occur when individuals learn about and form an impression of a new target item (Martin 1986; Martin and Achee 1992; Martin et al. 1990). This model begins with the premise that initial responses to new information are spontaneously assimilated to the context. Whether this effect of context is evident when judgments are expressed is posited to be a function of (1) whether contextual influence is perceived to be (in)appropriate to judgment and (2) the level of cognitive resources that the processor is able and willing to devote to correcting any such influence that is judged to be inappropriate (see Fig. 1a). Our findings both generalize and qualify this model, as illustrated in Figure $1 b$.

\section{Encoding Effects}

We generalize previous findings by demonstrating that a semantic connection between the context and target item is not required in order for affective contextual cues, such as a happy or sad mood, to influence initial reactions to new product information. However, the absence of any meaningful connection between the context and the product appears to affect the likelihood that correction for the contextual influence will occur. More specifically, when the context and the target are unrelated, the assessment that the contextual influence is inappropriate would seem to require relatively few cognitive resources. Consistent with this logic and in contrast with prior research (Martin et al. 1990; Meyers-Levy and Sternthal 1993), we find that even consumers who were not inclined to engage in thoughtful processing exhibited a contrast effect under certain conditions.

However, efforts to correct for inappropriate contextual influence may be constrained by other task demands that would seem to take precedence in drawing on the cognitive resources available. In particular, we observe that the resources required to identify the appropriate product category (i.e., the basic-level category) moderated the form of the context effect. When few resources were required to categorize the product, it appears that consumers had sufficient excess resources to partial out the effect of context, and overcorrection in this process resulted in a contrast effect. However, when categorization required substantial resources, it seems that consumers lacked the resources required to correct for contextual influence. As a result, the initial response of assimilation persisted.

These findings are consistent with the view that categorization precedes evaluation (Cohen and Basu 1987) and that it is resource demanding (Meyers-Levy and Tybout 1989; Ozanne, Brucks, and Gewal 1992). Further, these findings expand the role that categorization may play in determining context effects. Not only does categorization affect whether contextual influence is judged to be inappropriate by determining the overlap between the context and the target (e.g., Herr 1989; Meyers-Levy and Sternthal 1993), but the process of categorization may also influence the resources available to correct for contextual influence that is considered inappropriate.

Although the foregoing findings demonstrate the robustness of Martin's model, our work also introduces an important qualification of this model by identifying when the context effects that occur during encoding of new information may be absent at judgment. Our data suggest that such effects will be evident when either the nature of the judgment task or consumers' willingness to engage in effortful thought encourages retrieval of contextencoded information. More specifically, we found that feature judgments, for which the new product information would seem to be uniquely diagnostic, were consistently influenced by the context (i.e., context effects emerge independent of consumers' need for cognition). Context effects were also observed when more personal, overall reactions were requested of consumers whose need for cognition was high. Presumably these individuals' predisposition toward effortful thought led them to retrieve and to incorporate the encoded product information into their judgments regardless of whether other, context-independent information (e.g., category affect and prior experience), which might reasonably have been viewed as diagnostic for this type of judgment, was accessible.

In contrast, context effects were absent when consumers whose need for cognition was low expressed their overall evaluations. Apparently these individuals perceived alternative, context-independent sources of information to require less effort and to be sufficiently diagnostic of their personal reactions to the product. Although our procedure only revealed the absence of context effects and not the specific process by which these consumers formed their judgments, several possibilities are plausible. First, the process of categorizing the product may have made affect associated with the type of product (e.g., soft drink or fruit juice) accessible and this predetermined affect may have guided overall evaluations (see Simmons et al. [1993] for evidence that context effects may be absent when preformed judgments can be retrieved). Alternatively, the experience of tasting the product may have provided access to sensory data that were sufficiently removed from the context to be unaffected by it, and these data may have guided overall evaluations. Additional research is needed to disentangle the role played by these and other context-independent sources of affect when consumers whose need for cognition is low form their overall evaluations. However, we can conclude that although assimilation is commonly the default encoding strategy, this bias will not necessarily be evident at judgment when cognitive resources are limited.

\section{Integrating Encoding and Retrieval Effects}

Implicit in our discussion has been the assumption that the encoding context was no longer readily accessible at 
judgment. This assumption is consistent with our procedure, which included multiple tasks that would seem likely to interfere with continued rumination about the context, and it is supported by the observation that the favorableness of the context had no effect on the overall evaluations of low need for cognition consumers, who would seem likely to be influenced by the context-induced mood were it readily accessible.

However, there is ample evidence that when contextual cues are accessible at judgment, these cues may affect consumers' responses (see Schwarz and Bless [1992] for a review). Two ways in which the context at judgment may influence responses have been documented. The context may have a direct effect on judgments by providing relevant information. For example, one's current mood may be viewed as indicative of more general affective states, such as life satisfaction or happiness (Schwarz and Clore 1983), or it may be viewed as reflecting the effectiveness of an advertisement (Goldberg and Gorn 1987; Kamins et al. 1991; Yi 1990). In addition, accessible contextual cues may influence the subset of potentially relevant information that is retrieved and thus used in formatting a response (Schwarz and Bless 1992). Thus, being in a positive/negative mood may facilitate retrieval of knowledge that is affectively congruent with that mood in much the same way as we have posited occurs during encoding. To the extent that contextual information is included in the representation of the target at judgment, this information may be scrutinized for its appropriateness. When the context-related information is judged to be appropriate, its continued inclusion in the representation of the target is anticipated to result in an assimilation effect. By contrast, when the information retrieved is viewed as reflecting a contextual bias, efforts to partial out this influence are expected, and overcorrection, as well as changes in how the response scale is anchored, may produce a contrast effect (see Schwarz and Bless 1992). Thus, the process that is theorized to determine the type of context effect is quite similar regardless of whether this influence occurs at encoding or at judgment.

At the same time, the circumstances in which context effects attributable to encoding versus retrieval are likely to emerge appear to be somewhat different. Our findings reveal that encoding effects of context can occur in the absence of any meaningful connection between the context and the new information. Further, such effects may endure even though the context may no longer be accessible (i.e., context may have an indirect effect). However, these effects will only be reflected in judgments when either the task or the consumer's predisposition toward thoughtful processing encourage retrieval of the contextencoded information.

By contrast, only accessible contextual cues that have some substantive connection to the specific judgment requested appear to affect evaluations at the time a judgment is rendered (see Schwarz, Munkel, and Hippler 1990). Moreover, these effects, which are attributable to changes in the momentary representation of the target stimulus, are inherently unstable. Indeed, much of the attention that has focused on context effects at judgment would seem to be motivated by the desire to understand these biases so that they may be minimized, allowing pollsters to uncover more stable or "true" responses to the questions being posed.

Regardless of whether context has its influence at encoding or at retrieval, the models of context effects discussed to this point have assumed that consumers' initial response is one of assimilation. While this assumption is consistent with the vast majority of findings, recent evidence has emerged suggesting that contrast may sometimes be the default strategy. Petty and Wegener (1993) report that when the context activates extreme instances in the same category as the target object at judgment, the initial response reflects contrast, and efforts to correct for contextual influence may result in assimilation. Thus, Petty and Wegener propose a more general model of context effects, termed the flexible bias-correction model. This model allows the initial contextual influence to be one of either assimilation or contrast, and it emphasizes that it is the effort to correct for contextual influence, rather than contrast per se, that is resource demanding. The flexible correction perspective can be accommodated by the modified version of Martin's model that we propose (see Fig. 1b), and a fruitful line of future research would be to delineate more clearly the circumstances in which the initial reactions reflect assimilation versus contrast.

In sum, the present work both generalizes and qualifies Martin's (1986; Martin et al. 1990) model of context effects that occur during the encoding of information. We demonstrate that encoding effects of context can occur when the context and the target information lack any semantic connection. However, when a semantic connection is absent, it appears that little effort may be required to determine that the influence of the context is inappropriate and requires correction. As a result, individuals may seek to partial out contextual influence irrespective of their need for cognition. Whether correction actually occurs appears to be a function of the resources that individuals are able to devote to this task. In our experiments, when categorization of the product required substantial effort, the available resources were apparently insufficient for individuals also to correct for contextual influence. As a result, the initial response of assimilation persisted. When categorization was less effortful, however, sufficient resources could be devoted to the contextual data for correction to occur, and an overadjustment in this process produced a contrast effect. These effects of the context during encoding were reflected in subsequent judgments but only when either the nature of the judgment task or consumers' predisposition toward effortful thought encouraged retrieval of the context-encoded information. When such conditions were lacking, the context at encoding had no effect on judgments. Thus, in our revised version of Martin's model, cognitive resources available at encoding determine the type of contextual 
influence and cognitive resources devoted to judgment determine the likelihood that this influence will be evident in subsequent product evaluations.

\section{APPENDIX}

\section{Negative News Stories}

Joyce Williamson is all too familiar with the debilitating effects of depression. She has coped with bouts of depression on and off during her adult life and twice lost her job because of her inability to function when the depression hits. In trying to convey how the depression affects her, Williamson said, "When the depression comes over me, when I'm feeling really bad, even the smallest things in life such as making an error in my typing cause me great pain.',

Apparently Jill Paquette had a premonition that December 22 was not going to be a good day for her. That morning she told a coworker, "Somedays when I awake in the morning I know that nothing is going to go my way - and it doesn't.' Not only did her car break down on her way to work, requiring an expensive tow to the garage, but she returned to her apartment to discover that it had been robbed and all the Christmas gifts she had carefully bought and wrapped for her loved ones were gone.

\section{Positive News Stories}

Joyce Williamson is aware of how her mood affects interpretation of everyday events in her life. She tries to take a positive approach to her work and believes that this attitude has helped her to win two promotions in just one year on the job. Interviewed regarding her attitude and its effect on her work, Williamson said, "When I'm feeling good, when I really try to think positively, even the smallest things in life, such as completing a trivial task, are a source of pleasure.",

Apparently Jill Paquette had a premonition that December 22 was going to be a good day for her. That morning she told a coworker, "Some days when I awake in the morning I know that everything is going to go my wayand it does."' Traffic was lighter than usual so she got to the office early, turned on the radio and heard her name announced as the winner of WJCL's Christmas shopping spree. Not only will she have plenty of money for gifts for her loved ones as well as herself, the gifts will be wrapped and delivered free of charge.

[Received December 1991. Revised November 1996. John G. Lynch, Jr., served as editor and Deborah Roedder John served as associate editor for this article.]

\section{REFERENCES}

Allen, Chris T. and Chris A. Janiszewski (1989), “Assessing the Role of Contingency Awareness in Attitudinal Condi- tioning with Implications for Advertising Research,' Journal of Marketing Research, 30 (February), 30-43.

Bettman, James R., Noel Capon, and Richard J. Lutz (1975), "Multiattribute Measurement Models and Multiattribute Attitude Theory: A Test of Construct Validity,' Journal of Consumer Research, 1 (March), 1-15.

Cacioppo, John T. and Richard E. Petty (1982), "The Need for Cognition," Journal of Personality and Social Psychology, 42 (January), 116-131.

_ Richard E. Petty, and C. Kao (1984), “The Efficient Assessment of Need for Cognition,' Journal of Personality Assessment, 48 (June), 306-307.

Cohen, Joel B. and Kunal Basu (1987), “Alternative Models of Categorization: Toward a Contingent Processing Framework," Journal of Consumer Research, 13 (March), 455472.

Feldman, Jack M. and John G. Lynch, Jr. (1988), “Self-Generated Validity and Other Effects of Measurement on Belief, Attitude, Intention and Behavior,' Journal of Applied Psychology, 73 (August), 421-435.

Fishbein, Martin and Icek Ajzen (1975), Belief, Attitude, Intention and Behavior, Reading, MA: Addison-Wesley.

Fiske, Susan T. and Steven L. Neuberg (1990), “A Continuum of Impression Formation, from Category-Based to Individuating Processes: Influences of Information and Motivation on Attention and Interpretation,', Advances in Experimental Social Psychology, 23, 1-74.

Goldberg, Marvin E. and Gerald J. Gorn (1987), “Happy and Sad TV Programs: How They Affect Reactions to Commercials,' Journal of Consumer Research, 14 (December), 387-403.

Herr, Paul (1989), "'Priming Price: Prior Knowledge and Context Effects,' Journal of Consumer Research, 16 (June), $67-75$.

Hoch, Stephen J. and Young-Won Ha (1986), "Consumer Learning: Advertising and the Ambiguity of Product Experience,' Journal of Consumer Research, 13 (September), 221-233.

Kamins, Michael A., Lawrence J. Marks, and Deborah Skinner (1991), "Television Commercial Evaluation in the Context of Program Induced Mood: Congruency versus Consistency Effects,' Journal of Advertising, 20 (June), 1-14.

Kaplan, Martin F. (1976), "Measurement and Generality of Response Dispositions in Person Perception,' Journal of Personality, 44 (June), 179-194.

Lynch, John G., Dipankar Chakravarti, and Anusree Mitra (1991), "Contrast Effects in Consumer Judgments: Changes in Mental Representations or in the Anchoring of Rating Scales?', Journal of Consumer Research, 18 (December), 284-297.

Markus, Hazel and Robert B. Zajonc (1985), "The Cognitive Perspective in Social Psychology,"' in Handbook of Social Psychology, Vol. 1, ed. Gardner Lindzey and Elliot Aronson, New York: Random House, 137-230.

Martin, Leonard L. (1986), "Set/Reset: Use and Disuse of Concepts in Impression Formation,' Journal of Personality and Social Psychology, 51 (September), 493-504.

- and John W. Achee (1992), "Beyond Accessibility: The Role of Processing Objectives in Judgment,' in The Construction of Social Judgments, ed. Leonard L. Martin and Abraham Tesser, Hillsdale, NJ: Erlbaum.

— and Contrast as a Function of People's Willingness and 
Ability to Expend Effort in Forming an Impression,' Journal of Personality and Social Psychology, 59 (July), 2737.

Mathur, M. and Amitava Chattopadhyay (1991), “The Impact of Moods Generated by Television Programs on Responses to Advertising,', Psychology and Marketing, 8 (Spring), 59-77.

Meyers-Levy, Joan and Brian Sternthal (1993) "A Two-Factor Explanation of Assimilation and Contrast Effects,' Journal of Marketing Research, 30 (August), 359-368.

and Alice M. Tybout (1989), "Schema Congruity as a Basis for Product Evaluation,' Journal of Consumer Research, 16 (June), 39-54.

Ozanne, Julie L., Merrie Brucks, and Dhruv Gewal (1992), “A Study of Information Search Behavior during the Categorization of New Products,' Journal of Consumer Research, 18 (March), 452-463.

Park, Jong-Won and Manoj Hastak (1994), "Memory-Based Product Judgments: Effects of Involvement at Encoding and Retrieval,' Journal of Consumer Research, 21 (December), 534-547.

Petty, Richard E. and Duane T. Wegener (1993), “Flexible Correction Processes in Social Judgment: Correcting for Context-Induced Contrast,' Journal of Experimental Social Psychology, 29 (March), 137-165.

Rosch, Eleanor (1978), "Principles of Categorization," in Cognition and Categorization, ed. Eleanor Rosch and Barbara Lloyd, Hillsdale, NJ: Erlbaum, 27-48.

Schumann, David W. and Esther Thorson (1990), “'The Influence of Viewing Context on Commercial Effectiveness: A Selection-Processing Model,' in Current Issues and Research in Advertising, Vol. 12, ed. James H. Leigh and Claude R. Martin, Jr., Ann Arbor: University of Michigan Press, 1-24.
Schwarz, Norbert and Herbert Bless (1992), "Constructing Reality and Its Alternatives: An Inclusion/Exclusion Model of Assimilation and Contrast Effects in Social Judgment", in The Construction of Social Judgments, ed. Leonard L. Martin and Abraham Tesser, Hillsdale, NJ: Erlbaum, 217 245.

and Gerald L. Clore (1983), “Mood, Misattribution, and Judgments of Well-Being: Information and Directive Functions of Affective States,', Journal of Personality and Social Psychology, 45 (September), 513-523.

, T. Munkel, and H. J. Hippler (1990), "What Determines a 'Perspective'? Contrast Effects as a Function of the Dimension Tapped by Preceding Questions,' European Journal of Social Psychology, 20, 357-361.

Scott, Carol A. and Richard F. Yalch (1978), "A Test of the Self-Perception Explanation of the Effects of Reward on Intrinsic Interest,' Journal of Experimental Social Psychology, 14 (March), 180-192.

Shimp, Terence A., Elnora W. Stuart, and Randall W. Engle (1991), "A Program of Classical Conditioning Experiments Testing Variations in the Conditioned Stimulus and Context,' Journal of Consumer Research, 18 (June), 112.

Simmons, Carolyn J., Barbara A. Bickart, and John G. Lynch, Jr. (1993), "Capturing and Creating Public Opinion in Survey Research,' Journal of Consumer Research, 20 (September), 316-329.

Sujan, Mita and Christine Dekleva (1987), “Product Cetgorization and Inference Making: Some Implication for Comparative Advertising,' Journal of Consumer Research, 14 (December), 372-378.

Yi, Youjae (1990), "Cognitive and Affective Priming Effects of the Context for Print Advertisements,' Journal of Advertising, 19 (Summer), 40-48. 